\title{
Association of Cognitive Impairment in Patients on 3-Hydroxy-3-Methyl-Glutaryl-CoA Reductase Inhibitors
}

\author{
Satyajeet Roy, d, Joshua Louis Weinstock ${ }^{\mathrm{b}}$, Allyse Sachiko Ishino ${ }^{\mathrm{b}}$, Jefferson Felix Benites ${ }^{\mathrm{b}}$, \\ Samantha Rachel Pop ${ }^{\mathrm{b}}$, Christopher David Perez ${ }^{\mathrm{b}}$, Edvard Adrian Gumbs ${ }^{\mathrm{b}}$, Jennifer Ann Rosenbaum ${ }^{\mathrm{b}}$, \\ Mary Kate Roccato ${ }^{\mathrm{b}}$, Hely Shah ${ }^{\mathrm{b}}$, Gabriela Contino ${ }^{\mathrm{b}}$, Krystal Hunter $^{\mathrm{c}}$
}

\begin{abstract}
Background: Atherosclerotic cardiovascular diseases are the leading cause of death in the United States. A reduction in cholesterol with 3-hydroxy-3-methyl-glutaryl-CoA reductase inhibitors (statin) significantly reduces mortality and morbidity. Statins may be associated with cognitive impairment or dementia. Our aim was to study the association of cognitive impairment or dementia in patients who were on a statin.
\end{abstract}

Methods: Electronic medical records of 3,500 adult patients in our suburban internal medicine office were reviewed.

Results: There were $720(20.6 \%)$ patients in the statin treatment group. Dementia or cognitive impairment was an associated comorbid condition in $7.9 \%$ patients in the statin treatment group compared to $3.1 \%$ patients in the non-statin group $(\mathrm{P}<0.001)$. Analysis of all of the patients with cognitive impairment or dementia showed that among the age ranges of 51 years through 100 years, the patients in the statin treatment group had a higher prevalence of cognitive impairment or dementia compared to the non-statin group. In the statin treatment group, we found significantly higher prevalence of hyperlipidemia (86.3\%), hypertension $(69.6 \%)$, diabetes mellitus $(36.0 \%)$, osteoarthritis $(31.5 \%)$, coronary artery disease $(26.1 \%)$, hypothyroidism $(21.5 \%)$ and depression $(19.3 \%)$ compared to the non-statin group ( $\mathrm{P}<0.001)$. About $39.9 \%$ of the patients with dementia or $\operatorname{cog}$ nitive impairment were on statin therapy compared to $18.9 \%$ patients who had no dementia or cognitive impairment and were on statin therapy $(\mathrm{P}<0.001)$. Among the patients with cognitive deficit or dementia in the statin treatment group, the majority of the patients were either on atorvastatin $(43.9 \%)$ or simvastatin $(35.1 \%)$, followed by

Manuscript accepted for publication May 12, 2017

aDepartment of Medicine, Cooper University Hospital, Cooper Medical School of Rowan University, Camden, NJ, USA

${ }^{b}$ Cooper Medical School of Rowan University, Camden, NJ, USA

${ }^{c}$ Cooper Research Institute, Cooper Medical School of Rowan University, Camden, NJ, USA

${ }^{\mathrm{d} C}$ Corresponding Author: Satyajeet Roy, Department of Medicine, Cooper University Hospital, Cooper Medical School of Rowan University, 1103 North Kings Highway, Suite 203, Cherry Hill, NJ, USA.

Email: roy-satyajeet@cooperhealth.edu

doi: https://doi.org/10.14740/jocmr3066w rosuvastatin $(12.2 \%)$ and pravastatin $(8.8 \%)$. We found greater odds of dementia or cognitive impairment with each year increase in age (1.3 times), in women (2.2 times), African American race (2.7 times), non-consumption of moderate amount of alcohol (two times), diabetes mellitus (1.6 times), hypothyroidism (1.7 times), cerebrovascular accident (3.2 times), and other rheumatological diseases (1.8 times).

Conclusions: The association of dementia or cognitive impairment was significantly higher in the patients who were on statin therapy compared to the patients who were not on a statin.

Keywords: Cognitive function; Cognitive impairment; Dementia; Statin therapy; HMG-CoA reductase inhibitor therapy

\section{Introduction}

Atherosclerotic cardiovascular diseases (ASCVDs) include heart disease, stroke and other cardiovascular disorders. According to the current statistics, ASCVD is the underlying cause of one in every three deaths in the United States [1]. A reduction in the low-density lipoprotein cholesterol (LDL-C) has been shown to be associated with a relative risk reduction of $20-30 \%$ for myocardial infarction, about $20 \%$ for ischemic stroke and up to $15 \%$ for all-cause mortality related to ASCVD [2]. The 3-hydroxy-3-methyl-glutaryl-CoA (HMG-CoA) reductase inhibitors (commonly known as "statins") represent a group of medications that reduce cholesterol levels, especially the LDL-C. Long-term treatment with a statin is considered as safe and it improves survival in patients with ASCVD [3]. Additionally, long-term adherence to statin therapy is associated with progressively increasing clinical benefits in the form of primary and secondary prevention of atherosclerotic cardiovascular events [4].

Cholesterol is an integral component of the cell membrane signal transduction in the neurons and in the nerve endings. In a dry adult human brain, the total lipids in the gray matter, white matter and myelin constitute about $39.6 \%, 64.6 \%$ and $78.0 \%$ of dry weight, respectively [5].

Suboptimal lipid levels may potentially alter the composition and impair neuronal function. Several studies have suggested an association between the statins therapy, reduction in the circulating cholesterol levels and neurocognitive disorders 
Table 1. Baseline Characteristics

\begin{tabular}{|c|c|c|c|c|}
\hline Variable & All $(n=3,500)$ & $\begin{array}{l}\text { Group 1: statin treatment } \\
\text { group }(n=720)\end{array}$ & $\begin{array}{l}\text { Group 2: non-statin treatment } \\
\text { group }(n=2,780)\end{array}$ & P value (1 vs. 2) \\
\hline Age (years), mean (SD) & $46.9(17.6)$ & $65.8(14.3)$ & $46.9(16.5)$ & $<0.001 *$ \\
\hline \multicolumn{5}{|l|}{ Gender } \\
\hline Male (n, \%) & $1,590(45.4)$ & $405(56.2)$ & $1,185(42.6)$ & $<0.001^{\dagger}$ \\
\hline Female (n, \%) & $1,910(54.6)$ & $315(43.8)$ & $1,595(57.4)$ & $<0.001^{\dagger}$ \\
\hline African American (n, \%) & $473(13.5)$ & $74(10.3)$ & $399(14.4)$ & \\
\hline Hispanic (n, \%) & $326(9.3)$ & $50(6.9)$ & $276(9.9)$ & \\
\hline Other $(\mathrm{n}, \%)$ & $522(14.9)$ & $85(11.8)$ & $437(15.7)$ & \\
\hline \multicolumn{5}{|l|}{ Social factors } \\
\hline
\end{tabular}

*Wilcoxon two-sample test. †Fisher's exact test.

[6-11].

In 2014, the United States Food and Drug Administration (FDA) released its report on expanded adverse effects of statins [12]. According to the report memory loss, forgetfulness and confusion span all statin products and all age groups. This report was based on the review of the database and recorded reports of clinical trials on statins that included assessment of cognitive function $[6,7,11,13-21]$. The report also included the post-marketing adverse event reports of documented but reversible memory loss and cognitive impairment. The postmarketing adverse reports, reported via the Adverse Event Reporting System (AERS), upon which the FDA based its warnings, generally described individuals older than 50 years age who experienced ill-defined memory loss, confusion, and foggy thinking with variable onset of symptoms ranging from 1 day to years after statin exposure. The statins involved were primarily the lipophilic statins simvastatin and atorvastatin. These symptoms resolved after discontinuation of the statins and in some instances recurred with resumption. The reported cases did not appear to be associated with fixed or progressive dementia, such as Alzheimer's disease [12].

On the contrary, some studies have shown either no effect $[22,23]$, or neuroprotective benefits of statins for dementia or cognitive impairment [4, 24-29]. Similarly, hyperlipidemia and the sequelae of ASCVD are risk factors for dementia, hence according to other studies, it has been proposed that statins could play a role in protection from cognitive impairment [7]. Yet, reports of memory loss are mentioned in the second paragraph of the expanded advice to the consumers on statin risks reported by the FDA in the consumer health information [12]. The specific statin, dose, patient characteristics, and other comorbidities have not yet been determined by the available studies. The objective of our retrospective study was to determine the association of cognitive impairment and dementia in patients in our clinical practice who were on a statin.

\section{Materials and Methods}

\section{Study selection}

This study was a retrospective electronic medical record review that observed the association between a multitude of factors and cognitive impairment in patients who were on statin and compared with the patients who were not on statin. Patients who were seen between July 1, 2015 and December 31,2015 were included in this study. The study was reviewed and approved by the Institutional Review Board of the Cooper Health System, Camden, New Jersey, USA. The inclusion criterion was adult patients of age 18 years or older who were either on statin therapy, or not. The exclusion criterion was patients under the age of 18 years.

\section{Data collection}

The following data were collected for each patient: age, gender, race (Caucasian, African American, Hispanic or other), social history (tobacco use, alcohol use, and/or recreational drug use), diagnosis of cognitive deficit or dementia, family history of dementia, comorbid medical conditions, such as hypertension, hyperlipidemia, hypothyroidism, coronary artery disease, cerebral vascular attack, carotid artery stenosis, congestive heart failure, chronic obstructive pulmonary disease, asthma, chronic kidney disease, liver disease, HIV, other immunodeficiencies, post-organ transplant immunodeficiency, cancer, other endocrine disorders, psychiatric conditions (depression, bipolar, anxiety disorder, and schizophrenia), arthritis and other rheumatologic diseases. We also collected systolic and diastolic blood pressures, body mass index, statin use, name of the statin, statin dose, and medication for dementia. Measure- 


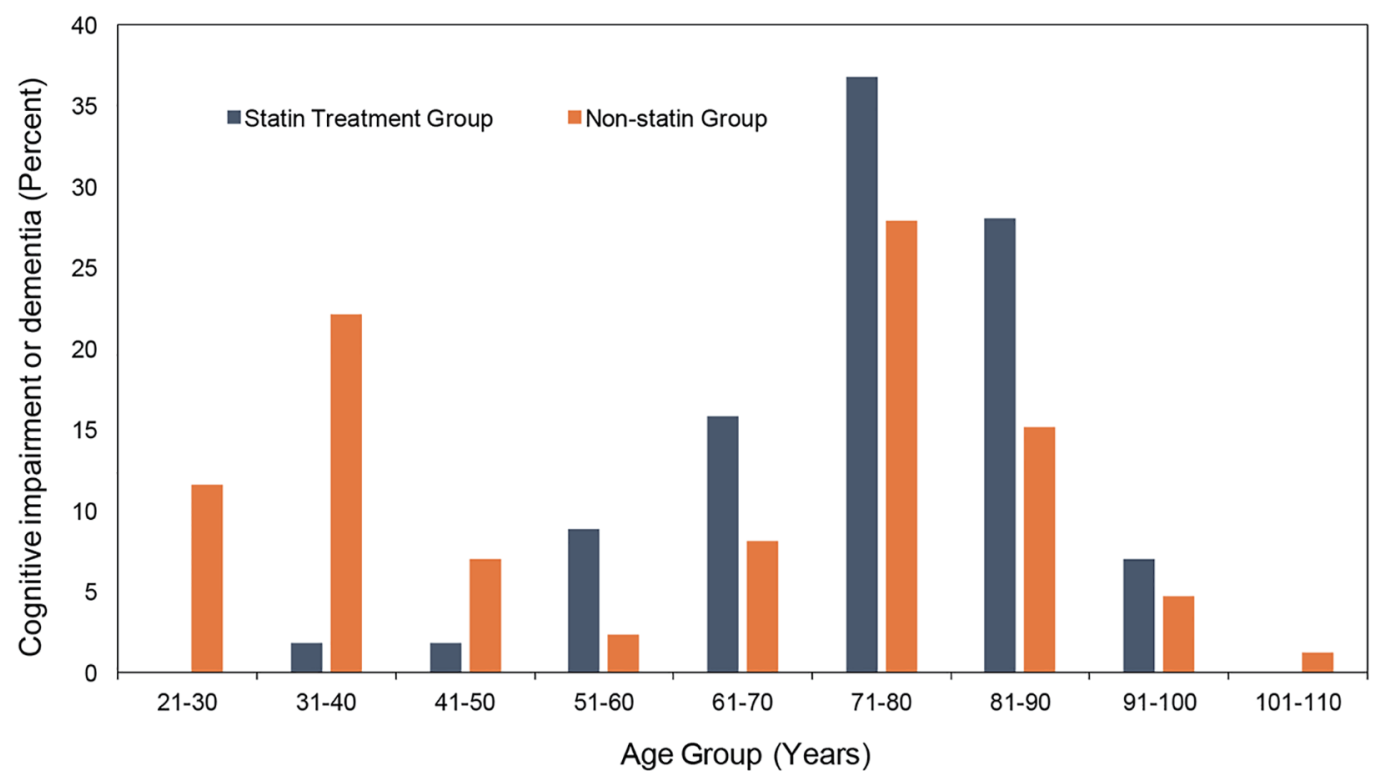

Figure 1. Comparative frequency of all cases of cognitive impairment or dementia based on age group.

ments of total cholesterol, triglycerides, high-density lipoprotein cholesterol (HDL-C), and LDL-C were also included in this study.

\section{Statistical analysis}

Collected data were entered into a Microsoft Excel (2013, Redmond, WA, USA) spreadsheet. Statistical analysis was done using SPSS (Statistical Package for the Social Sciences, version 15.01, IBM, Armonk, NY, USA). Subjects were divided into two groups. Group 1 represented the patients who were on statin (statin treatment group). Group 2 represented the patients who were not on statin (non-stain group). Baseline group characteristics of the study population were compared using univariate analysis and multivariate analysis. For univariate analysis, the independent $t$-test or Mann-Whitney $\mathrm{U}$ test was used to compare continuous variables between the two study groups and between those with diagnosis of cognitive deficit or dementia and those who did not have cognitive deficit or dementia. Chi-square tests were used to compare categorical variables between the groups. For multivariate analysis, logistic regression was used. The dependent variable was cognitive deficit or dementia and the independent variable was statin use. Other independent variables in the model were determined by the univariate analysis. Those with a $\mathrm{P}$ value of 0.20 or less were included in the regression model. In this study, significance was defined as a $\mathrm{P}<0.05$.

\section{Results}

A total of 3,500 patients were included in the study. The age range of our study population was 19 - 103 years. There were $720(20.6 \%)$ patients who were on a statin (group 1: statin treatment group). The remaining 2,780 (79.4\%) patients were not on a statin (group 2: non-statin group).

The mean age of the patients in the statin treatment group was $65.8 \pm 14.3$ years and in the non-statin group was $46.9 \pm$ 16.5 years. The difference in the mean age was statistically significant $(\mathrm{P}<0.001)$ (Table 1$)$.

There were significantly more male patients $(56.2 \%)$ compared to the female patients $(43.8 \%)$ in the statin treatment group $(\mathrm{P}<0.001)$. Moreover, among all of the male patients in our study, there were significantly more male patients $(56.2 \%)$ in the statin treatment group than in the non-statin group (42.6\%) $(\mathrm{P}<0.001)$.

The race analysis showed that the majority of the patients in the statin treatment group were Caucasian (71.0\%), followed by African American (10.3\%), other race (11.8\%) and Hispanic $(6.9 \%)$ (Table 1). We found similar hierarchy of the frequency of race in the non-statin group as well, but there were significantly more Caucasians in the statin treatment group $(71.0 \%)$ compared to the non-statin group $(60.0 \%)(\mathrm{P}<0.001)$, while there were significantly less African Americans, other races and Hispanics in the statin treatment group $(10.3 \%, 11.8 \%$ and $6.9 \%$, respectively) compared to the non-statin group $(14.4 \%$, $15.7 \%$ and $9.9 \%$, respectively) $(\mathrm{P}<0.001)$ (Table 1$)$.

The analysis of the social factors such as alcohol intake, cigarette smoking and use of recreational drugs was observed in $47.3 \%, 37.4 \%$, and $4.5 \%$ of all patients, respectively. Intergroup comparison showed no statistically significant difference in alcohol use or recreational drug use between the statin treatment group and the non-statin group, but there were significantly more patients in the statin treatment group $(50.5 \%)$ compared to the non-statin group $(34.0 \%)$ who smoked cigarettes $(\mathrm{P}<0.001)$ (Table 1$)$.

Dementia or cognitive impairment was identified as an associated comorbid condition in $7.9 \%$ patients in the statin treatment group compared to $3.1 \%$ patients in the non-statin 
Table 2. Associated Comorbid Conditions

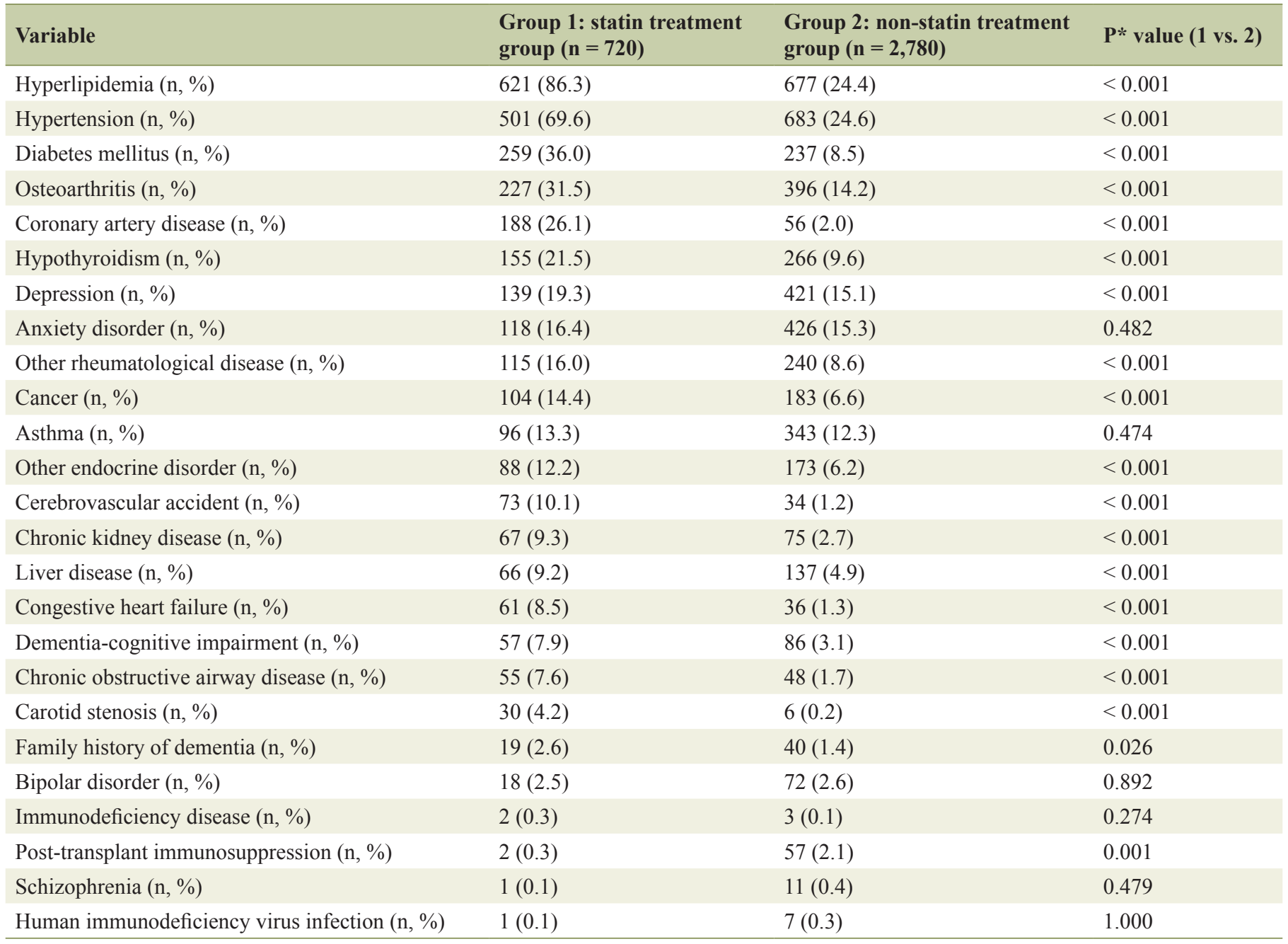

${ }^{*}$ Fisher's exact test.

group. The difference was statistically significant $(\mathrm{P}<0.001)$.

Further analysis of all of the patients with cognitive impairment or dementia showed that among the age ranges of 51 years through 100 years, the patients in the statin treatment group had a higher prevalence of cognitive impairment or dementia compared to the non-statin group (Fig. 1). We found a higher prevalence of cognitive impairment or dementia in the younger population (ages 21 - 50) due to cognitive deficits associated with childhood onset developmental disorders, such as mental retardation, cerebral palsy, autism spectrum disorder and other childhood onset cognitive disorders (Fig. 1). Although there was a higher prevalence of family history of dementia in the statin treatment group $(2.6 \%)$ compared to the non-statin group (1.4\%), the difference was not statistically significant $(\mathrm{P}=0.026)$.

In the statin treatment group, more than two-thirds of the patients had hyperlipidemia $(86.3 \%)$ and hypertension (69.6\%), about one-third had diabetes mellitus (36.0\%) and osteoarthritis (31.5\%), about a quarter had coronary artery disease (26.1\%), and about one-fifth had hypothyroidism (21.5\%) and depression (19.3\%). The prevalence of all of these associated comorbid conditions was significantly higher in the statin treatment group than in the non-statin group $(\mathrm{P}<0.001)(\mathrm{Ta}-$ ble 2, Fig. 2). Similarly, patients in the statin treatment group had a significantly higher prevalence of other rheumatological disorders (12.2\%), cerebrovascular accidents (10.1\%), chronic kidney disease $(9.3 \%)$, liver disease $(9.2 \%)$, congestive heart failure $(8.5 \%)$, chronic obstructive airway disease $(7.6 \%)$ and carotid stenosis $(4.2 \%)$ compared to the non-statin group (Table 2). The prevalence of other associated comorbid conditions, such as anxiety disorder, asthma, bipolar disorder, immunodeficiency disease, post-transplant immunosuppression, schizophrenia and HIV infection was comparable in both the groups.

The majority of the patients $(n=511$, or $70.9 \%)$ in the statin treatment group were in the age range of $51-80$ years (Fig. $3)$. This subgroup was further analyzed and compared with the patients in the age group of 51 - 80 years in the non-statin group (Table 3 ). The mean age of the patients in this subgroup of patients who were in the age range of 51 - 80 years and were 


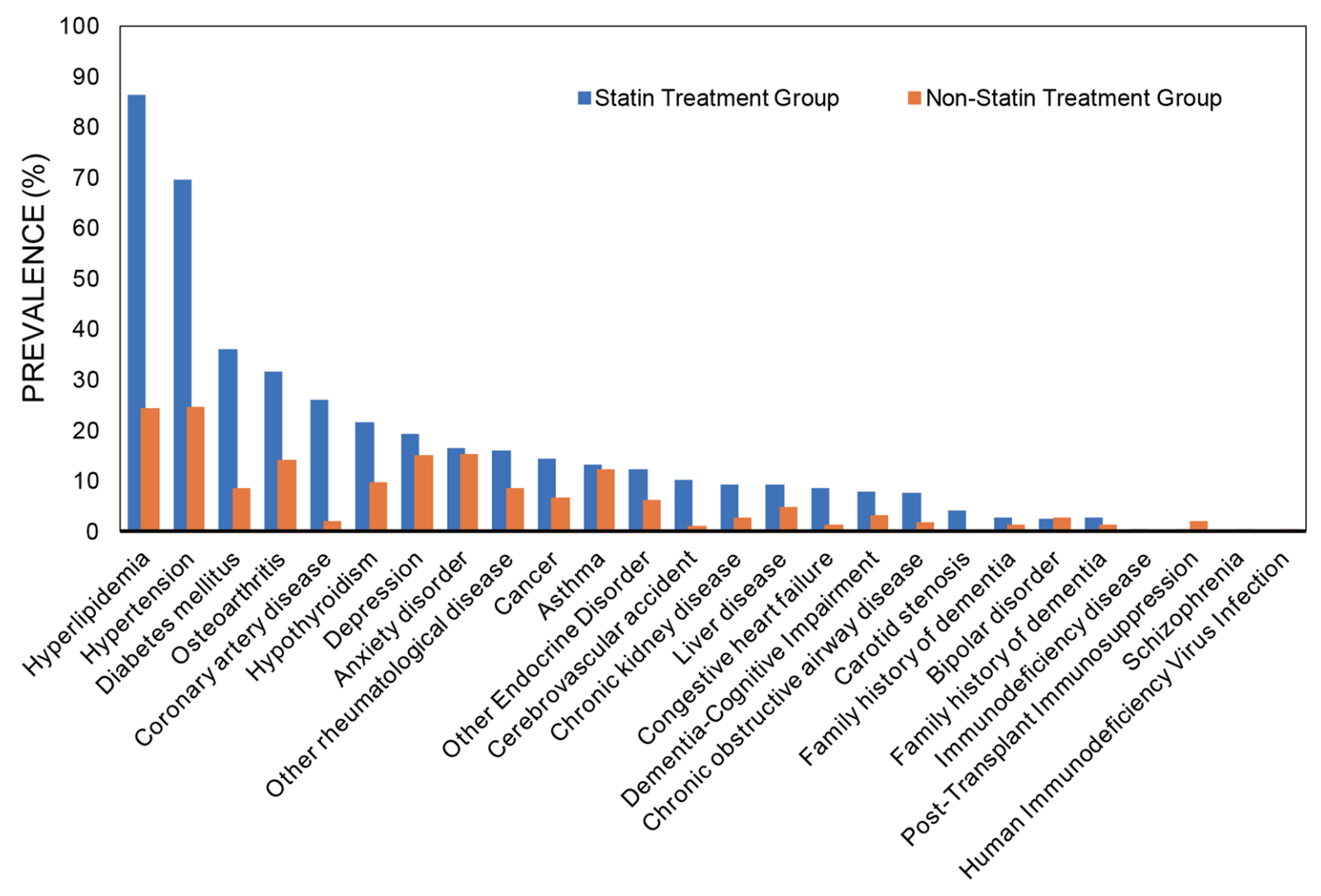

ASSOCIATED COMORBID CONDITIONS

Figure 2. Associated comorbid conditions.

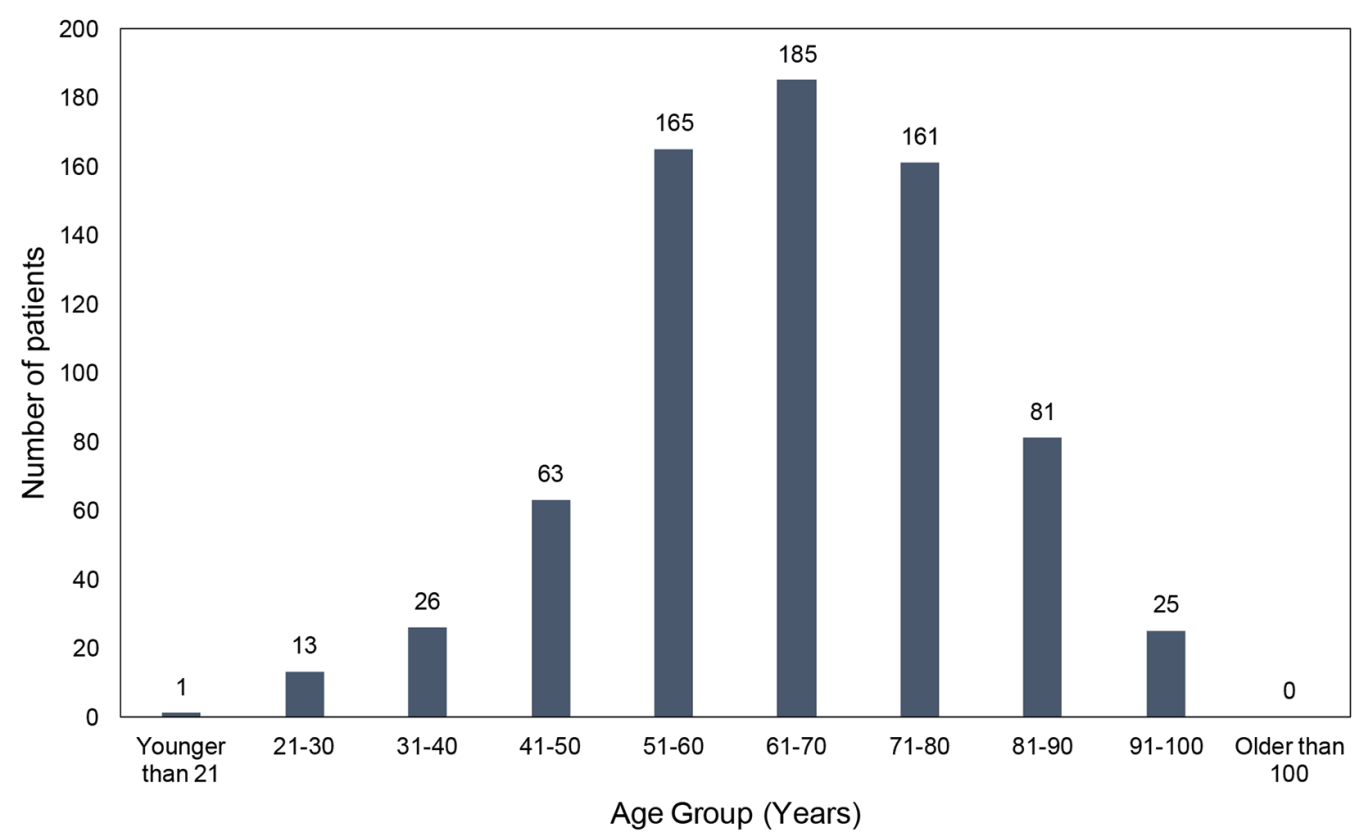

Figure 3. Age distribution of the patients in the statin treatment group. 
Table 3. Associated Comorbid Conditions in the 51 - 80 Years Age Subgroup

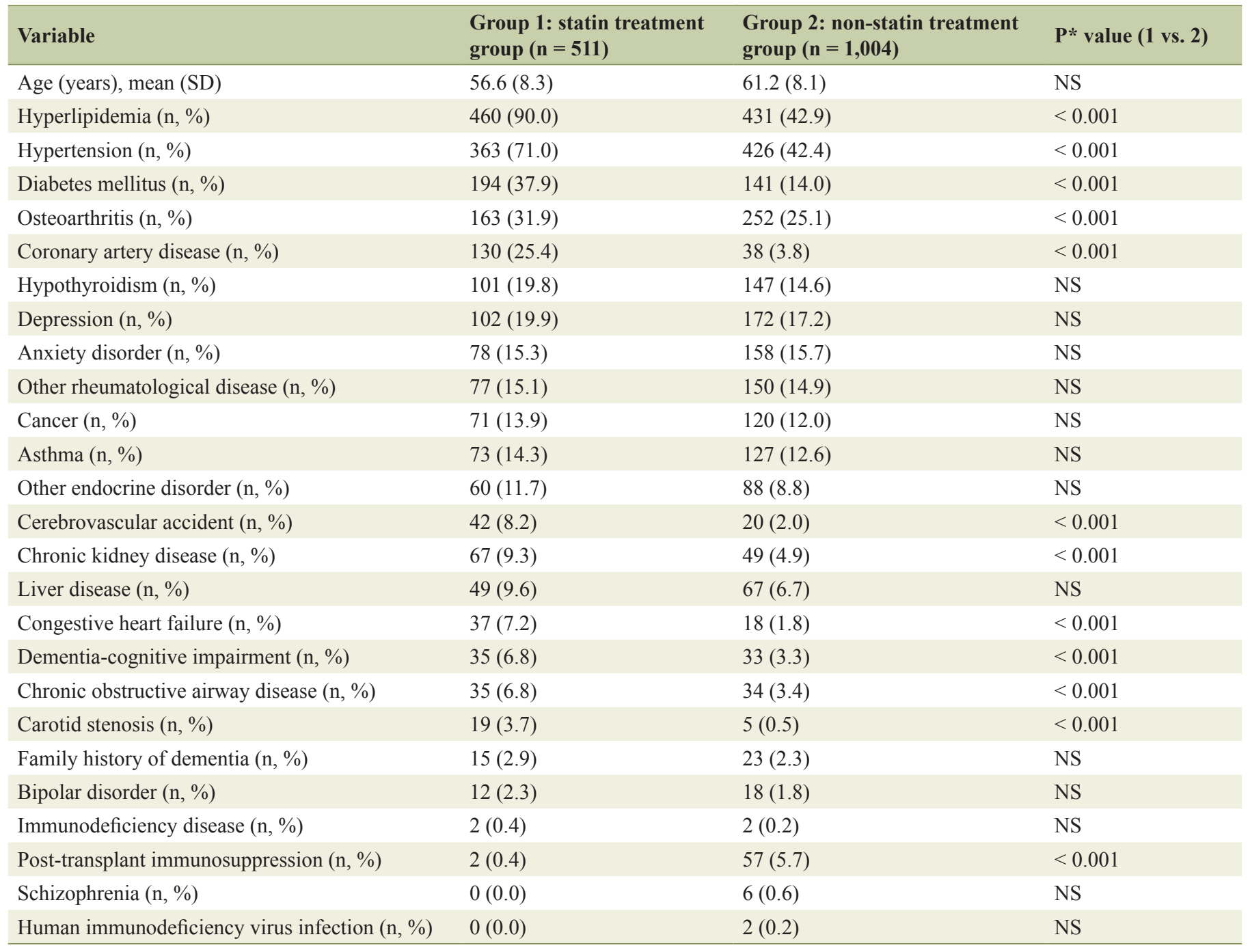

*Fisher's exact test. NS: not significant.

Table 4. Comparative Vitals and Lipid Analysis

\begin{tabular}{|c|c|c|c|}
\hline Variable & $\begin{array}{l}\text { Group 1: statin treatment } \\
\text { group }(n=720)\end{array}$ & $\begin{array}{l}\text { Group 2: non-statin treatment } \\
\text { group }(n=2,780)\end{array}$ & $P *$ value (1 vs. 2) \\
\hline \multicolumn{4}{|l|}{ Vitals } \\
\hline BMI $\left(\mathrm{kg} / \mathrm{m}^{2}\right)$, mean $(\mathrm{SD})$ & $29.7(6.5)$ & $29.5(10.9)$ & 0.556 \\
\hline SBP (mm Hg), mean (SD) & $127.4(14.9)$ & $123(13.7)$ & $<0.001$ \\
\hline DBP (mm Hg, mean (SD) & $75.9(9.6)$ & $76.5(9.1)$ & 0.141 \\
\hline TG (mg/dL), mean (SD) & $136.1(102.3)$ & $113.4(70.8)$ & $<0.001$ \\
\hline HDL-C (mg/dL), mean (SD) & $51.2(15.8)$ & $54.5(17.9)$ & $<0.001$ \\
\hline LDL-C (mg/dL), mean (SD) & $98.0(40.8)$ & $108.2(30.8)$ & $<0.001$ \\
\hline
\end{tabular}

BMI: body mass index; SBP: systolic blood pressure; DBP: diastolic blood pressure; TC: total cholesterol; TG: triglyceride; HDL-C: high-density lipoprotein cholesterol; LDL-C: low-density lipoprotein cholesterol. *Wilcoxon two-sample test. 
Table 5. Statin Therapy and Dementia or Cognitive Impairment

\begin{tabular}{llll}
\hline Variable & $\begin{array}{l}\text { Dementia or cognitive } \\
\text { impairment }(\mathbf{n}=\mathbf{1 4 3})\end{array}$ & $\begin{array}{l}\text { No dementia or cognitive } \\
\text { impairment }(\mathbf{n}=\mathbf{3}, \mathbf{3 5 7})\end{array}$ & $\mathbf{P}$ \\
\hline Statin therapy $(\mathrm{n}, \%)$ & $57(39.9)$ & $662(18.9)$ & $<0.001$ \\
\hline
\end{tabular}

in the statin treatment group was $56.6 \pm 8.3$ years and in the non-statin group was $61.2 \pm 8.1$ years. Although the mean age of the patients in this subgroup of patients who were in the statin treatment group was lower than subgroup of the patients in the non-statin group, the difference was not statistically significant (Table 3). The prevalence of cognitive impairment or dementia among the patients who were in the age range of 51 - 80 years was $6.8 \%$ in the statin treatment group and $3.3 \%$ in the non-statin group (Table 3 ). The difference was statistically significant $(\mathrm{P}<0.001)$. Although there was a higher prevalence of ASCVD defining conditions (such as coronary artery disease, cerebrovascular accidents, carotid artery stenosis and congestive heart failure), ASCVD risk factors (such as hyperlipidemia, hypertension and diabetes mellitus), and certain comorbid conditions (such as osteoarthritis, chronic obstructive pulmonary disease and chronic kidney disease), none of the factors can be directly attributed as a cause of cognitive impairment resulting in a higher prevalence of cognitive deficit or dementia in the statin treatment group. Additionally, there was no difference in the prevalence of certain known risk factors for cognitive impairment or dementia between the two groups, such as hypothyroidism, liver disease, other endocrine disorders and family history of dementia (Table 3 ).
The mean (SD) systolic blood pressure was higher in the statin treatment group $(127.4 \pm 14.9)$ compared to the nonstatin group $(123 \pm 13.7)(\mathrm{P}<0.001)($ Table 4$)$. There were no statistically significant differences in the mean body mass indices and diastolic blood pressures between the two groups (Table 4). In the statin treatment group, the mean total cholesterol, LDL-C and HDL-C were lower (176.5, 98.0 and $51.2 \mathrm{mg} / \mathrm{dL}$, respectively) compared to the non-statin group (185.1, 108.2 and $54.5 \mathrm{mg} / \mathrm{dL}$, respectively). All the differences were statistically significant $(\mathrm{P}<0.001)$. The mean triglyceride level was higher $(136.1 \mathrm{mg} / \mathrm{dL})$ in the statin treatment group compared to the non-statin group $(113.4 \mathrm{mg} / \mathrm{dL})(\mathrm{P}<0.001)$ (Table 4$)$.

Further analysis of the association of statin therapy and dementia or cognitive impairment showed that $39.9 \%$ of patients with dementia or cognitive impairment were on statin therapy compared to $18.9 \%$ patients who had no dementia or cognitive impairment and were on statin therapy. The difference was statistically significant $(\mathrm{P}<0.001)$ (Table 5). Among the patients with cognitive deficit or dementia in the statin treatment group, the majority of the patients were either on atorvastatin $(43.9 \%)$ or simvastatin $(35.1 \%)$, followed by rosuvastatin $(12.2 \%)$ and pravastatin (8.8\%) (Fig. 4).

The retrospective review of the medical records provided

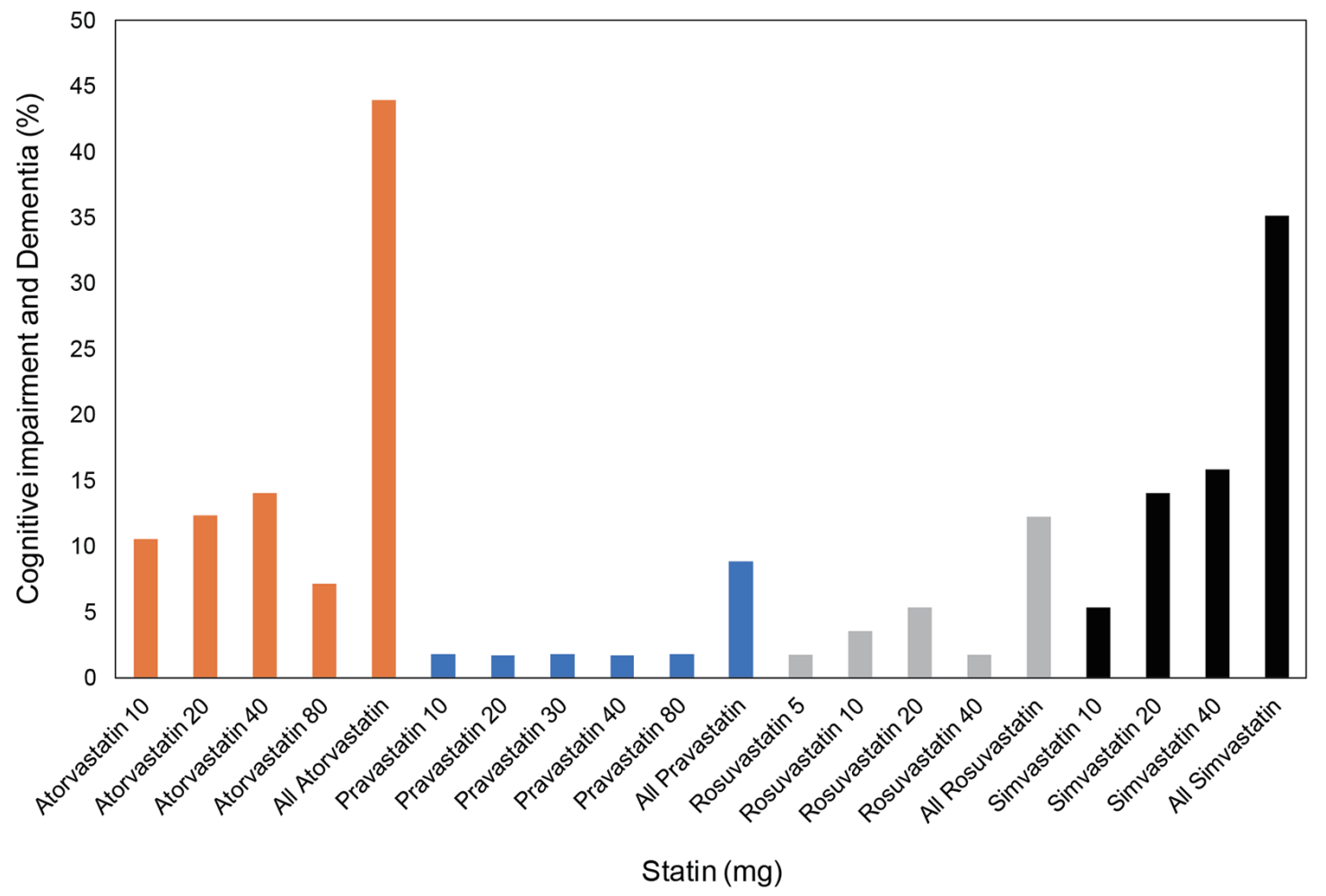

Figure 4. Type of statin and cognitive impairment or dementia. 
periodic documentation of lipid levels without specific date of diagnosis of hyperlipidemia or date of statin initiation for a specific statin treatment group. In our institution, the electronic medical records were implemented in 2008. Patient data were manually entered from previous paper charts without specific mention of the onset of the statin therapy or cognitive impairment. Patients with cognitive impairment or dementia were either treated with donepezil, memantine or rivastigmine. All of the patients in the statin treatment group remained on statin at the time of data acquisition, hence there was no documentation of cessation of statin therapy and changes in cognitive function status. Diagnostic documentation of cognitive impairment or dementia was based on mini-mental status examination and/ or Montreal cognitive assessment test. We also reviewed the possible negative cognitive influence of psychoactive medications in patients who had psychiatric disorders, such as depression, anxiety, bipolar disorder and/or schizophrenia. We found comparable prevalence of depression, anxiety, bipolar disorder and/or schizophrenia between the patients in the statin treatment group and in the non-statin treatment group who were in the age range of $51-80$ years (Table 3 ).

Additionally, we looked into the factors that might have contributed to dementia or cognitive impairment and performed a multivariate analysis. We found that the odds of getting dementia or cognitive impairment increased by $1.3 \%$ with each year increase in age. Women had 2.2 times greater odds of having dementia or cognitive impairment. African American patients had 2.7 times greater odds of having dementia or cognitive impairment than other minorities who were non-Hispanic. Patients who did not consume moderate amount of alcohol had two times increased odds of having dementia or cognitive impairment than those who consumed alcohol. We found an opposite relationship between the diastolic blood pressure and dementia or cognitive impairment. The average diastolic blood pressure was lower in patients with dementia or cognitive impairment than those without. Patients with diabetes mellitus had 1.6 times greater odds of having dementia or cognitive impairment. Similarly, patients with hypothyroidism had 1.7 times greater odds of having dementia or cognitive impairment, patients with cerebrovascular accident had 3.2 times greater odds of having dementia or cognitive impairment, and patients with other rheumatological diseases had 1.8 times greater odds of having dementia or cognitive impairment.

\section{Discussion}

Our study had three major findings: first, a significantly higher association of dementia or cognitive impairment as a comorbid condition in patients who were on statin therapy compared to the patients who were not on statin therapy; second, a significantly higher number of patients with dementia or cognitive impairment were on statin therapy compared to the patients without dementia or cognitive impairment; third, atorvastatin and simvastatin were the leading statins associated with dementia or cognitive impairment.

Our findings were supported by several studies that have found a similar association between statins and cognitive impairment and other psychological disorders, such as an increased risk of suicide [6-11].

Muldoon and colleagues compared the changes in performance between lovastatin-treated patients and placebo. They found statistically significant differences in the tests of attention and psychomotor speed indicating greater improvement in the placebo group compared to the lovastatin-treated patients [11]. Another study by Muldoon and colleagues showed that patients on statins demonstrated lack of otherwise expected normal improvements on repeated tests of attention and reaction time taken over the course of 6 months compared with placebo. This study was subsequently focused on simvastatin which partially supported that patients on simvastatin had minor decrements in cognitive function [6]. A study by Evans and Golomb showed negative cognitive effects with statins, and the effects were directly related to the potency of the statins. It offered significant negative impact in the quality of life [7]. According to the 2014 Food and Drug Administration (FDA) report memory loss, forgetfulness and confusion span all statin products across all age groups [12]. A review by Wagstaff and colleagues concluded that statins may be associated with cognitive impairment but they could not determine a causal relationship [14].

Our observation of $7.9 \%$ prevalence of cognitive impairment or dementia in our patients who were on a statin corresponds with the findings of Glasser and colleagues who found $8.6 \%$ prevalence of cognitive impairment in statin users in a national cohort study of the United States population in which 7,191 participants were on a statin and 17,404 participants were not on statins [30]. It is important to mention that adverse drug reactions due to statins are less likely discussed with the patients by the physicians, unless the patients initiate the discussion. One study found that muscle related adverse effects were the most likely statin related adverse effect discussed, while the cognitive changes were rarely assessed, evaluated or initiated by the physicians [31]. It is fair to estimate that voluntary reporting of adverse event of cognitive impairment due to statins has been variable and mostly under-reported. This might underestimate the prevalence of cognitive impairment associated with the statins.

Kessler and colleagues found that healthcare providers do not think to report adverse events to the FDA that might be associated with medications [32]. Various studies indicate that the percentage of voluntary reporting to the AERS varies between $1 \%$ and $10 \%$ which is considered to be extremely low reporting [32]. Sahebzamani and colleagues found 2,566 reports of cognitive impairment for atorvastatin and simvastatin documented in AERS over a period of 8 years. They further extrapolated the data based on underreporting assuming $1-10 \%$ of reporting and concluded that about $3,000-30,000$ cognitive impairments each year may be associated with simvastatin and atorvastatin [8].

Several mechanisms have been explained between the association of cognitive impairment and statins. Ayiniyet and colleagues postulate that there happens to be a connection between low serum cholesterol and lower activity of central serotonergic release which influences the mood, cognition and impulsive behavior [9]. Engelberg reviewed available physio- 
logical mechanism behind low cholesterol levels and cognitive deficit [33]. It has been observed in the animal models that increase in the brain synaptosomal membrane cholesterol results into a robust increase in the number of serotonin receptors that are responsible for the maintenance of adequate cognitive and behavioral response. Lowering the synaptosomal membrane cholesterol with statins decreases the number of serotonin receptors, which in turn decreases synaptic binding and uptake of serotonin resulting into reduced cognitive and behavioral response [33].

We found that dementia or cognitive impairment was observed in a significantly higher proportion with the lipophilic statins, such as atorvastatin and simvastatin (79\%) compared to the hydrophilic statins, such as rosuvastatin and pravastatin $(21 \%)$. Our findings were supported by many studies that have shown higher association and severity of cognitive deficit with lipophilic statins compared with hydrophilic statins $[8,14,34-$ 38].

Thelen and colleagues reported significant reduction in the brain cholesterol precursor levels in simvastatin-treated mice (simvastatin $100 \mathrm{mg} / \mathrm{kg}$ body weight) compared to hydrophilic pravastatin or controls after 3 days of oral intake [35]. A study by Sahebzamani and colleagues showed a significantly higher proportion of cognitive impairment associated with the commonly prescribed lipophilic statins, atorvastatin and simvastatin, compared to hydrophilic statins, such as rosuvastatin and pravastatin [8].

Studies suggest that hyperlipidemia inhibits intracellular cholesterol synthesis in the neurons and increases intracellular amyloid $(\mathrm{Ab})$ production which results into cell lysis which facilitates inhibition of cholesterol synthesis. Lipophilic statins, such as simvastatin and atorvastatin, further inhibit neuronal cholesterol which exacerbates neuronal degeneration resulting into cognitive impairment and dementia [36]. In a study, Chauhan and colleagues found that lipophilic statin, such as lovastatin significantly increased cerebral levels of IL-1beta and TNF-alpha in the animal model suggesting an enhanced cerebral inflammatory mechanism which may be responsible for the alteration in the function of the neurons [39]. Lipophilic statins cross the blood-brain barrier which may influence the synthesis of cholesterol in the neurons [40]. Reduction in the supply of unesterified cholesterol in the nerve cells affects cell integrity and function. Lipophilic statins diffuse via both passive diffusion and non-selective diffusion into hepatocytes and non-hepatocyte tissues [41-46]. On the contrary, hydrophilic statins are highly hepatoselective and enter into the hepatocytes via active transportation, which makes them less likely associated with non-hepatocytic adverse effects [36, 42]. Among the lipophilic statins, the likelihood of associated adverse effects predominantly depend upon their mechanisms of action.

The most commonly prescribed lipophilic statins atorvastatin and simvastatin are metabolized through CY3PA4. Atorvastatin has a longer half-life (about 11 - $30 \mathrm{~h}$ ) while simvastatin has a higher percentage of absorption (about 65-85\%). Other lipophilic statins, such as fluvastatin go through an extensive first-pass metabolism and protein binding. This may explain why atorvastatin and simvastatin may have higher association with non-hepatocytic adverse effects (such as cognitive impairment and dementia) among all of the lipophilic statins
$[36,42]$.

Rojas-Fernandez and colleagues analyzed the impact of statin use on cognition. According to their analysis in suspected individuals with statin-induced cognitive impairment switching from lipophilic to hydrophilic statins might resolve cognitive impairment [37].

It is important to mention that there have been many observational studies, randomized studies and reviews that have found no association between statins and cognitive impairment or dementia [47-50].

Other notable findings in our study were a higher association of hyperlipidemia, diabetes mellitus, hypertension and other cardiovascular risk factors or end-organ damages in the statin treatment group. It is appropriate to say that the patients in the statin treatment group fell into a higher ASCVD risk category, hence they were on a statin. It is also important to mention that although we found a higher association of depression in the statin treated patients, nevertheless we did not observe a higher association of other psychological disorders, such as anxiety disorder, bipolar disorder and schizophrenia in the statin treatment group. Our findings do not fully correlate with the previously reported studies [9-11]. Our multivariate analysis identified certain risk categories with higher odds of having cognitive impairment or dementia, such as increasing age, women, African American ethnicity, non-consumption of moderate amount of alcohol, lower diastolic blood pressure, diabetes mellitus, hypothyroidism, cerebrovascular accidents, and rheumatological diseases other than osteoarthritis. Our findings correspond with many studies which support our observation on increasing age [51-53], women [54], African American ethnicity [55], non-consumption of moderate amount of alcohol [56], lower diastolic blood pressure [57], diabetes mellitus [58], hypothyroidism [59-62], cerebrovascular accidents [63], and rheumatological diseases other than osteoarthritis [64, 65].

The major strength of our study was a large sample size from one office location. Patients' subsequent visits with the same healthcare provider allowed somewhat active recognition and documentation of cognitive changes in the electronic medical record. Nevertheless, our study had several limitations, such as retrospective analysis of only documented variables, and analysis limited to a suburban outpatient population, which cannot be generalized.

We conclude that association of dementia or cognitive impairment was significantly higher in our patients who were on statin therapy compared to the patients who were not on a statin.

\section{Acknowledgments}

The authors thank Christine Rickette RN (study coordinator) for her contribution to this study.

\section{Conflicts of Interest}

None. 


\section{Grant Support}

None.

\section{References}

1. Mozaffarian D, Benjamin EJ, Go AS, Arnett DK, Blaha MJ, Cushman M, Das SR, et al. Heart Disease and Stroke Statistics-2016 Update: A Report From the American Heart Association. Circulation. 2016;133(4):e38-360.

2. Baigent C, Keech A, Kearney PM, Blackwell L, Buck G, Pollicino C, Kirby A, et al. Efficacy and safety of cholesterol-lowering treatment: prospective meta-analysis of data from 90,056 participants in 14 randomised trials of statins. Lancet. 2005;366(9493):1267-1278.

3. Randomised trial of cholesterol lowering in 4444 patients with coronary heart disease: the Scandinavian Simvastatin Survival Study (4S). Lancet. 1994;344(8934):13831389.

4. Simpson RJ, Jr., Mendys P. The effects of adherence and persistence on clinical outcomes in patients treated with statins: a systematic review. J Clin Lipidol. 2010;4(6):462471.

5. O'Brien JS, Sampson EL. Lipid composition of the normal human brain: gray matter, white matter, and myelin. J Lipid Res. 1965;6(4):537-544.

6. Muldoon MF, Ryan CM, Sereika SM, Flory JD, Manuck SB. Randomized trial of the effects of simvastatin on cognitive functioning in hypercholesterolemic adults. Am J Med. 2004;117(11):823-829.

7. Evans MA, Golomb BA. Statin-associated adverse cognitive effects: survey results from 171 patients. Pharmacotherapy. 2009;29(7):800-811.

8. Sahebzamani FM, Munro CL, Marroquin OC, Diamond DM, Keller E, Kip KE. Examination of the FDA Warning for Statins and Cognitive Dysfunction. J Pharmacovigilance. 2014;2(141):3-9.

9. Ainiyet J, Rybakowski J. [Low concentration level of total serum cholesterol as a risk factor for suicidal and aggressive behavior]. Psychiatr Pol. 1996;30(3):499-509.

10. Kaplan JR, Muldoon MF, Manuck SB, Mann JJ. Assessing the observed relationship between low cholesterol and violence-related mortality. Implications for suicide risk. Ann N Y Acad Sci. 1997;836:57-80.

11. Muldoon MF, Barger SD, Ryan CM, Flory JD, Lehoczky JP, Matthews KA, Manuck SB. Effects of lovastatin on cognitive function and psychological well-being. Am J Med. 2000;108(7):538-546.

12. U.S. Food and Drug Administration. Consumer Health Information. FDA expands advice on statin risks. 2014. Available at: https://www.acc.org/ /media/Non-Clinical/.../1\%20FDA\%20StatinRisk0114.pdf. Accessed April 26, 2017

13. Orsi A, Sherman O, Woldeselassie Z. Simvastatin-associated memory loss. Pharmacotherapy. 2001;21(6):767769 .

14. Wagstaff LR, Mitton MW, Arvik BM, Doraiswamy PM.
Statin-associated memory loss: analysis of 60 case reports and review of the literature. Pharmacotherapy. 2003;23(7):871-880.

15. Parker BA, Polk DM, Rabdiya V, Meda SA, Anderson K, Hawkins K, Pearlson GD, et al. Changes in memory function and neuronal activation associated with atorvastatin therapy. Pharmacotherapy. 2010;30(6):236e-240e.

16. Zamrini E, McGwin G, Roseman JM. Association between statin use and Alzheimer's disease. Neuroepidemiology. 2004;23(1-2):94-98.

17. Beydoun MA, Beason-Held LL, Kitner-Triolo MH, Beydoun HA, Ferrucci L, Resnick SM, Zonderman AB. Statins and serum cholesterol's associations with incident dementia and mild cognitive impairment. J Epidemiol Community Health. 2011;65(11):949-957.

18. Bettermann K, Arnold AM, Williamson J, Rapp S, Sink $\mathrm{K}$, Toole JF, Carlson MC, et al. Statins, risk of dementia, and cognitive function: secondary analysis of the ginkgo evaluation of memory study. J Stroke Cerebrovasc Dis. 2012;21(6):436-444

19. Benito-Leon J, Louis ED, Vega S, Bermejo-Pareja F. Statins and cognitive functioning in the elderly: a population-based study. J Alzheimers Dis. 2010;21(1):95-102.

20. Trompet S, van Vliet P, de Craen AJ, Jolles J, Buckley BM, Murphy MB, Ford I, et al. Pravastatin and cognitive function in the elderly. Results of the PROSPER study. J Neurol. 2010;257(1):85-90.

21. Feldman HH, Doody RS, Kivipelto M, Sparks DL, Waters DD, Jones RW, Schwam E, et al. Randomized controlled trial of atorvastatin in mild to moderate Alzheimer disease: LEADe. Neurology. 2010;74(12):956-964.

22. McGuinness B, O'Hare J, Craig D, Bullock R, Malouf R, Passmore P. Statins for the treatment of dementia. Cochrane Database Syst Rev. 2010;8:CD007514.

23. Richardson K, Schoen M, French B, Umscheid CA, Mitchell MD, Arnold SE, Heidenreich PA, et al. Statins and cognitive function: a systematic review. Ann Intern Med. 2013;159(10):688-697.

24. Corrao G, Ibrahim B, Nicotra F, Zambon A, Merlino L, Pasini TS, Catapano AL, et al. Long-term use of statins reduces the risk of hospitalization for dementia. Atherosclerosis. 2013;230(2):171-176.

25. Li G, Larson EB, Sonnen JA, Shofer JB, Petrie EC, Schantz A, Peskind ER, et al. Statin therapy is associated with reduced neuropathologic changes of Alzheimer disease. Neurology. 2007;69(9):878-885.

26. Vance JE. Dysregulation of cholesterol balance in the brain: contribution to neurodegenerative diseases. Dis Model Mech. 2012;5(6):746-755.

27. Jick H, Zornberg GL, Jick SS, Seshadri S, Drachman DA. Statins and the risk of dementia. Lancet. 2000;356(9242):1627-1631.

28. Kurata T, Miyazaki K, Kozuki M, Panin VL, Morimoto $\mathrm{N}$, Ohta $\mathrm{Y}$, Nagai $\mathrm{M}$, et al. Atorvastatin and pitavastatin improve cognitive function and reduce senile plaque and phosphorylated tau in aged APP mice. Brain Res. 2011;1371:161-170.

29. Wolozin B, Kellman W, Ruosseau P, Celesia GG, Siegel G. Decreased prevalence of Alzheimer disease associated 
with 3-hydroxy-3-methyglutaryl coenzyme A reductase inhibitors. Arch Neurol. 2000;57(10):1439-1443.

30. Glasser SP, Wadley V, Judd S, Kana B, Prince V, Jenny N, Kissela B, et al. The association of statin use and statin type and cognitive performance: analysis of the reasons for geographic and racial differences in stroke (REGARDS) study. Clin Cardiol. 2010;33(5):280-288.

31. Golomb BA, McGraw JJ, Evans MA, Dimsdale JE. Physician response to patient reports of adverse drug effects: implications for patient-targeted adverse effect surveillance. Drug Saf. 2007;30(8):669-675.

32. Kessler DA. Introducing MEDWatch. A new approach to reporting medication and device adverse effects and product problems. JAMA. 1993;269(21):2765-2768.

33. Engelberg H. Low serum cholesterol and suicide. Lancet. 1992;339(8795):727-729.

34. Golomb BA, Evans MA, Dimsdale JE, White HL. Effects of statins on energy and fatigue with exertion: results from a randomized controlled trial. Arch Intern Med. 2012;172(15):1180-1182.

35. Thelen KM, Rentsch KM, Gutteck U, Heverin M, Olin M, Andersson U, von Eckardstein A, et al. Brain cholesterol synthesis in mice is affected by high dose of simvastatin but not of pravastatin. J Pharmacol Exp Ther. 2006;316(3):1146-1152.

36. Sparks DL, Connor DJ, Browne PJ, Lopez JE, Sabbagh MN. HMG-CoA reductase inhibitors (statins) in the treatment of Alzheimer's disease and why it would be ill-advise to use one that crosses the blood-brain barrier. J Nutr Health Aging. 2002;6(5):324-331.

37. Rojas-Fernandez $\mathrm{CH}$, Cameron JC. Is statin-associated cognitive impairment clinically relevant? A narrative review and clinical recommendations. Ann Pharmacother. 2012;46(4):549-557.

38. Locatelli S, Lutjohann D, Schmidt HH, Otto C, Beisiegel $\mathrm{U}$, von Bergmann K. Reduction of plasma 24S-hydroxycholesterol (cerebrosterol) levels using high-dosage simvastatin in patients with hypercholesterolemia: evidence that simvastatin affects cholesterol metabolism in the human brain. Arch Neurol. 2002;59(2):213-216.

39. Chauhan NB, Siegel GJ, Feinstein DL. Effects of lovastatin and pravastatin on amyloid processing and inflammatory response in TgCRND8 brain. Neurochem Res. 2004;29(10):1897-1911.

40. Heverin M, Meaney S, Lutjohann D, Diczfalusy U, Wahren J, Bjorkhem I. Crossing the barrier: net flux of 27-hydroxycholesterol into the human brain. J Lipid Res. 2005;46(5):1047-1052.

41. Kim MC, Ahn Y, Jang SY, Cho KH, Hwang SH, Lee MG, Ko JS, et al. Comparison of clinical outcomes of hydrophilic and lipophilic statins in patients with acute myocardial infarction. Korean J Intern Med. 2011;26(3):294303.

42. Hamelin BA, Turgeon J. Hydrophilicity/lipophilicity: relevance for the pharmacology and clinical effects of HMG-CoA reductase inhibitors. Trends Pharmacol Sci. 1998;19(1):26-37.

43. Schachter M. Chemical, pharmacokinetic and pharmacodynamic properties of statins: an update. Fundam Clin
Pharmacol. 2005;19(1):117-125.

44. Pfefferkorn JA, Song Y, Sun KL, Miller SR, Trivedi BK, Choi C, Sorenson RJ, et al. Design and synthesis of hepatoselective, pyrrole-based HMG-CoA reductase inhibitors. Bioorg Med Chem Lett. 2007;17(16):4538-4544.

45. Corsini A, Bellosta S, Baetta R, Fumagalli R, Paoletti R, Bernini F. New insights into the pharmacodynamic and pharmacokinetic properties of statins. Pharmacol Ther. 1999;84(3):413-428.

46. White CM. A review of the pharmacologic and pharmacokinetic aspects of rosuvastatin. J Clin Pharmacol. 2002;42(9):963-970.

47. Bays H. Statin safety: an overview and assessment of the data - 2005. Am J Cardiol. 2006;97(8A):6C-26C.

48. Law M, Rudnicka AR. Statin safety: a systematic review. Am J Cardiol. 2006;97(8A):52C-60C.

49. Brass LM, Alberts MJ, Sparks L. An assessment of statin safety by neurologists. Am J Cardiol. 2006;97(8A):86C$88 \mathrm{C}$.

50. Rojas-Fernandez CH, Goldstein LB, Levey AI, Taylor BA, Bittner V, The National Lipid Association's Safety Task F. An assessment by the Statin Cognitive Safety Task Force: 2014 update. J Clin Lipidol. 2014;8(3 Suppl):S5-16.

51. Langa KM, Levine DA. The diagnosis and management of mild cognitive impairment: a clinical review. JAMA. 2014;312(23):2551-2561.

52. Kurz A, Diehl J, Riemenschneider M, Perneczky R, Lautenschlager N. [Mild cognitive disorder. Questions of definition, diagnosis, prognosis and therapy]. Nervenarzt. 2004;75(1):6-15.

53. Plassman BL, Langa KM, Fisher GG, Heeringa SG, Weir DR, Ofstedal MB, Burke JR, et al. Prevalence of cognitive impairment without dementia in the United States. Ann Intern Med. 2008;148(6):427-434.

54. McCarrey AC, An Y, Kitner-Triolo MH, Ferrucci L, Resnick SM. Sex differences in cognitive trajectories in clinically normal older adults. Psychol Aging. 2016;31(2):166-175.

55. Potter GG, Plassman BL, Burke JR, Kabeto MU, Langa KM, Llewellyn DJ, Rogers MA, et al. Cognitive performance and informant reports in the diagnosis of cognitive impairment and dementia in African Americans and whites. Alzheimers Dement. 2009;5(6):445-453.

56. Kim JW, Lee DY, Lee BC, Jung MH, Kim H, Choi YS, Choi IG. Alcohol and cognition in the elderly: a review. Psychiatry Investig. 2012;9(1):8-16.

57. Novak V, Hajjar I. The relationship between blood pressure and cognitive function. Nat Rev Cardiol. 2010;7(12):686-698

58. Roy S, Kim N, Desai A, Komaragiri M, Baxi N, Jassil N, Blessinger $\mathrm{M}$, et al. Cognitive Function and Control of Type 2 Diabetes Mellitus in Young Adults. N Am J Med Sci. 2015;7(5):220-226.

59. Breteler MM, van Duijn CM, Chandra V, Fratiglioni L, Graves AB, Heyman A, Jorm AF, et al. Medical history and the risk of Alzheimer's disease: a collaborative re-analysis of case-control studies. EURODEM Risk Factors Research Group. Int J Epidemiol. 1991;20(Suppl 2):S36-42. 
60. Erlanger DM, Kutner KC, Jacobs AR. Hormones and cognition: current concepts and issues in neuropsychology. Neuropsychol Rev. 1999;9(4):175-207.

61. Osterweil D, Syndulko K, Cohen SN, Pettler-Jennings PD, Hershman JM, Cummings JL, Tourtellotte WW, et al. Cognitive function in non-demented older adults with hypothyroidism. J Am Geriatr Soc. 1992;40(4):325-335.

62. van Boxtel MP, Menheere PP, Bekers O, Hogervorst E, Jolles J. Thyroid function, depressed mood, and cognitive performance in older individuals: the Maastricht Aging Study. Psychoneuroendocrinology. 2004;29(7):891-898.
63. Sun JH, Tan L, Yu JT. Post-stroke cognitive impairment: epidemiology, mechanisms and management. Ann Transl Med. 2014;2(8):80.

64. Maradit-Kremers H, Crowson CS, Nicola PJ, Ballman KV, Roger VL, Jacobsen SJ, Gabriel SE. Increased unrecognized coronary heart disease and sudden deaths in rheumatoid arthritis: a population-based cohort study. Arthritis Rheum. 2005;52(2):402-411.

65. Cordoliani-Mackowiak MA, Henon H, Pruvo JP, Pasquier F, Leys D. Poststroke dementia: influence of hippocampal atrophy. Arch Neurol. 2003;60(4):585-590. 